\title{
Estado nutricional, niveles de actividad \\ física y factores de riesgo cardiovascular \\ en estudiantes de la Universidad Santo Tomás
}

\author{
Nutritional status, levels of physical \\ activity and cardiovascular risk factors in \\ college students of Santo Tomas University
}

\begin{abstract}
Introduction: Non-communicable chronic diseases (NCCD) increase the presence of risk factors (RF) of cardiovascular disease (CVD) in young people. Objective: To analyze the relation among the nutritional status (NS), physical activity (PA) and the prevalence of cardiovascular RF in students from Santo Tomas University in Temuco enrolled in year 2010. Subjects and methods: A cross-sectional descriptive study was carried out; 177 out of 348 students of both genders were chosen as the sample, $32.2 \%$ males and $68.8 \%$ females. The age ranged between 18 and 25 years old. Anthropometric and blood pressure measurements, blood samples, PA surveys and obesity backgrounds were taken. The study outcomes helped to estimate the NS, the practice of PA, the prevalence of the different RF. Outcomes: The study showed that the $34.5 \%$ of the students present malnutrition by excess, $70.6 \%$ are sedentary, and a $9.6 \%$ is at risk of CVD by presence of metabolic syndrome (MS). The risk by abdominal obesity reached $53.1 \%$. Parameters which showed significant differences were HDL cholesterol lowered in active students ( $p=$ 0.027). When the PA together with BMI variables, triglycerides, LDL cholesterol, glycaemia and blood pressure were analyzed, no significant differences were observed. Conclusions: A high prevalence of malnutrition by excess, for being sedentary and $R F$ of CVD was present with a predominance in female students. Key words: Risk factors; cardiovascular diseases; obesity; students.
\end{abstract}

\section{INTRODUCCIÓN}

La encuesta nacional de salud (ENS) realizada en los años 2009-2010 reportó elevadas prevalencias de exceso de peso $(64,5 \%)$, sedentarismo $(88,6 \%)$ y síndrome metabólico (35\%) (1). Se conoce que existe una clara relación entre los factores de riesgo (FR) y los estilos de vida (2), los cuales se asocian a las enfermedades cardiovasculares $(E C V)$, patología que actualmente es la principal causa de muerte en personas adultas (3) y en países con niveles de desarrollo más elevados (4), generando un grave problema de salud pública (5). En Chile, producto de la transición demográfica y epidemiológica de las últimas décadas, la situación es similar $(6,7)$, su emergencia como problema de salud pública fue resultado de cambios sociales y económicos que modificaron el estilo de vida de un gran porcentaje de la población (8).
Manuel Alarcón H. (1)

Pedro Delgado F. $(2,4)$

Felipe Caamaño N. (3)

Aldo Osorio P. (4)

Marcelo Rosas M. (1)

Freddy Cea L. (1)

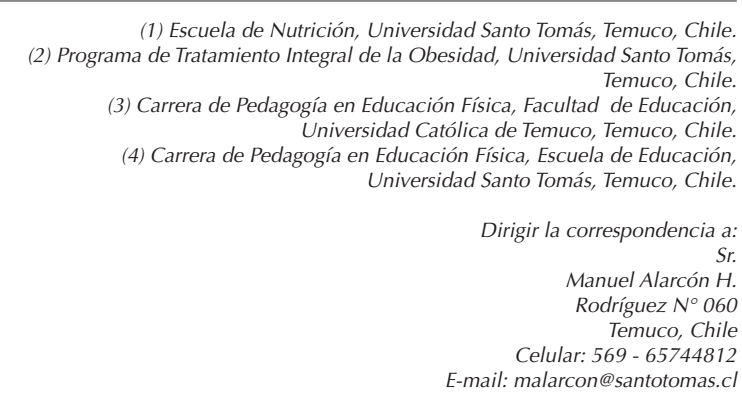

Este trabajo fue recibido el 29 de Abril de 2014 y aceptado para ser publicado el 29 de Noviembre de 2014.

Chile actualmente tiene una esperanza de vida elevada; 82 años para mujeres y 76 años para hombres (9), pero presenta una alta tasa de factores de riesgo predisponentes al desarrollo de enfermedades crónicas, las que muestran una prevalencia elevada en el adulto, aumentando la mortalidad prematura $(10,11)$, lo que fundamenta la preocupación que tienen los gobiernos en la prevención, manejo y control de los principales riesgos para reducir la carga de esta enfermedad, lo que podría reducir también el costo económico que significan para las personas y el Estado (12).

Los estudiantes universitarios se encuentran en una etapa del ciclo vital clave para la adopción de estilos de vida, que practicarán en el ámbito familiar, social y laboral. Por lo que se deben aportar datos consistentes sobre los factores de riesgo cardiovascular (FRCV) en los jóvenes, evitando sus consecuencias, y además debe ser un primer destino de los 
esfuerzos de prevención para reducir las enfermedades cardiovasculares (13-16).

El objetivo del presente estudio fue analizar la relación entre estado nutricional (EN), actividad física (AF) y prevalencia de FRCV en estudiantes de la Universidad Santo Tomás, sede Temuco, Chile.

\section{SUJETOS Y MÉTODO}

Se trata de un estudio cuantitativo, descriptivo de corte transversal, con análisis de FRCV, de un total de 348 estudiantes que ingresaron a la Universidad Santo Tomás el año 2010. Se obtuvo una muestra de 177 sujetos mediante un muestreo aleatorio simple. El tamaño muestral se calculó con un nivel de confianza de $95 \%$ y un error de $5 \%$.

Este estudio fue aprobado por el Comité de Ética de la Universidad Santo Tomás.

Recolección de la información: Los estudiantes fueron citados a una charla donde se expusieron los objetivos de la investigación, quienes decidieron participar del estudio firmaron un consentimiento informado.

Los antecedentes familiares y personales de ECV, el hábito tabáquico y los niveles de actividad física se determinaron mediante la aplicación del cuestionario validado de la II Encuesta Nacional de Calidad de Vida y Salud (ECV 2006) (17), se utilizaron sólo las preguntas que tenían relación con la investigación. Se consideró activo a quien realizaba actividad física como mínimo 3 veces por semana, 30 minutos cada vez. El hábito tabáquico se consideró en aquella persona que ha fumado 1 o más cigarrillos en el último mes, y que ha consumido más de 100 cigarrillos en su vida.

La medición de la presión arterial (PA) cumplió con el procedimiento de la guía clínica de hipertensión arterial primaria o esencial en personas de 15 años y más (18).

El peso se determinó con una balanza calibrada de palanca de adulto mecánica de columna $220 \mathrm{~kg}$, graduada, marca $S E C A \circledast$, y la talla se determinó con un tallímetro marca SECA®, graduada en $\mathrm{mm}$. Parar calcular el IMC se utilizó el índice de Quetelet. El estado nutricional se clasificó como: IMC normal = 18,5 - 24,9 kg/m2; sobrepeso 25,0 a 29,9 kg/ $\mathrm{m} 2$ y obesidad mayor o igual a $30 \mathrm{~kg} / \mathrm{m} 2$.

El contorno de cintura (CC) se determinó con una cinta métrica autorretráctil para adultos graduada en centímetros de marca SECA®. La medición utilizó el punto medio entre la espina ilíaca anterosuperior y el margen costal inferior, al final de una espiración normal, promediándose dos mediciones, para hombres se consideró obesidad $>88 \mathrm{~cm}$ y para mujeres $>83 \mathrm{~cm}$.

La evaluación de los parámetros bioquímicos se realizó mediante muestras sanguíneas recolectadas en tubos sin anticoagulante. Las muestras se centrifugaron a 2500 r.p.m. por 10 minutos, para obtener el suero a ser usado en las determinaciones bioquímicas. Las concentraciones séricas de glicemia (GL), colesterol total (Col-Total), colesterol HDL (HDL), colesterol LDL (LDL) y triglicéridos (TG). Se determinaron por métodos colorimétrico estándares, las que fueron medidas en el autoanalizador HumaStar80. La concentración de LDL se obtuvo mediante la fórmula de Friedwald, cuando la concentración de triglicéridos fue inferior a $400 \mathrm{mg} / \mathrm{dL}$. Estas muestras fueron extraídas por tecnólogos médicos. Las muestras fueron tomadas y procesadas en el Laboratorio de Bioquímica de la Universidad Santo Tomás.

El síndrome metabólico se determinó según la presencia y sumatoria de, al menos tres factores de riesgo presentes en cada estudiante. Los parámetros considerados fueron: Perímetro abdominal, hombres $\geq 88 \mathrm{~cm}$ y mujeres $\geq 83 \mathrm{~cm}$; TG $\geq 150 \mathrm{mg} / \mathrm{dl}$; HDL, hombres < $40 \mathrm{mg} / \mathrm{dl}$ y mujeres < $50 \mathrm{mg}$ / dl; presión arterial, $\geq 130 / 85$ o en tratamiento y glicemia en ayunas, $\geq 100 \mathrm{mg} / \mathrm{dl}$.

Análisis estadístico: Se realizó un análisis de corte exploratorio para depurar la información, determinando la prevalencia de las principales variables de estudio en conjunto con un análisis descriptivo. Posterior a ello se procedió a normalizar aquellas variables que no presentaban un comportamiento normal, a través de Log $\mathrm{N}$ y/o rangeo de datos. Para la comparación de las variables paramétricas cuantitativas entre dos grupos se utilizó la prueba t de Student, y cuando existían más de dos se realizó un ANOVA. En el caso de variables no paramétricas, se utilizó la prueba de la U de Mann-Whitney cuando se comparaban dos variables, y la prueba de la $\mathrm{H}$ de Kruskal- Wallis, para comparar más de dos variables. Todos los análisis se realizaron con el programa SPSS, versión 11,5 para Windows. El nivel de confianza fue del $95 \%$, $(p<0,05)$.

\section{RESULTADOS}

El $32,2 \%$ de la muestra correspondió a hombres y $68,8 \%$ a mujeres, $34,5 \%$ de los estudiantes presentaba exceso de peso, de los cuales $28,3 \%$ correspondió a sobrepeso y $6,2 \%$ a obesidad. El 29, 4\% de los estudiantes eran activos y el 70,6 $\%$ clasificaba como sedentario. En los parámetros metabólicos destaca que los valores de HDL se mostraron disminuidos en $51,4 \%$. En tanto, la frecuencia de otros parámetros bioquímicos, como el nivel de LDL se encontró aumentado en 44,1\%, mientras 35\% presentó un Col-total sobre los 200 mg/ dL. Una menor proporción de los estudiantes presentó tres o cuatro FRCV asociados y se clasificaron con presencia de SM $(9,6 \%)$ (tabla 1$)$.

Se apreciaron valores de triglicéridos estadísticamente diferentes, mayores en hombres, de los niveles, glicemia y en la presión arterial sistólica, el HDL presenta niveles superiores en mujeres, en cambio los valores promedio de IMC, contorno cintura, colesterol total y LDL, no mostraron diferencias significativas al relacionar los FRCV y la variable sexo (tabla 2).

Al relacionar la presencia de $S M$ con los distintos FRCV, se observó una diferencia significativa con las variables antropométricas, IMC, CC, HDL, GL y presión arterial. En tanto el Col-Total, TG y el nivel de LDL no presentaron diferencias entre la población estudiada con SM y aquellos que no clasificaban en esta categoría (tabla 3).

Los parámetros que evidenciaron diferencias significativas al relacionarlas con la AF fueron el HDL que se mostró disminuido en los estudiantes activos, y el Col-total que se observó levemente aumentado entre los sedentarios. Al relacionar la AF con las variables IMC, CC, TG, LDL y GL no se observaron diferencias estadísticamente significativas (tabla 4).

Al analizar la relación entre la obesidad visceral y otros FR (tabla 5), se observó que los estudiantes con mediciones superiores a lo normal de CC (mujer $\geq 83 \mathrm{~cm}$ - hombre $\geq 88$ $\mathrm{cm}$ ) son los que presentaban diferencias significativas con un IMC más elevado, mayor concentración de Col-total, GL, LDL y PA diastólica.

Los estudiantes que presentaban malnutrición por exceso, son quienes presentan mayor medición de CC, esta relación fue significativa, misma relación se observó entre los niveles de LDL y la medición de PA sistólica y diastólica. Por el con- 
trario, no se evidenciaron diferencias entre el diagnóstico de EN y el comportamiento de los parámetros de HDL, CT, TC y GL (tabla 6).

\section{DISCUSIÓN}

De los diferentes $\mathrm{FR}$, el de mayor proporción es el sedentarismo, que alcanzó 70,6\%, con claro predominio de las mujeres por sobre los hombres ( $80 \%$ y $49 \%$ respectivamente). Esta tendencia se repite en jóvenes de la universidad Austral de Valdivia donde se estimó en $87 \%$ la población sedentaria (19), pero se presenta como una cifra muy superior al com- parar con estudiantes mexicanos que alcanzan un 33,8\% (20).

En los estudiantes clasificados como sedentarios se evidenciaron valores mayores que en otra población con bajo nivel de actividad física en; Col-Total y LDL y menor en HDL (21). El sedentarismo es un factor de riesgo modificable, no sólo de ECV, sino además de otras enfermedades crónicas $(22,23)$. Se debe promover la práctica del ejercicio en la población, hoy en día existen estrategias costo-efectivos tales como los protocolos HIIT, que mejoran la capacidad metabólica del miocito (24).

Los resultados del estudio revelaron que $9,6 \%$ del total

TABLA 1

Estado nutricional, sedentarismo y presencia de factor de riesgo cardiovascular de los estudiantes, según género.

\begin{tabular}{|c|c|c|c|c|c|c|}
\hline \multirow[t]{2}{*}{ Estado Nutricional } & \multicolumn{2}{|c|}{ Hombre } & \multicolumn{2}{|c|}{ Mujer } & \multicolumn{2}{|c|}{ Total } \\
\hline & $\mathrm{n}$ & $\%$ & $\mathrm{~N}$ & $\%$ & $\mathrm{n}$ & $\%$ \\
\hline Enflaquecido & 2 & 3,5 & 3 & 2,5 & 5 & 2,8 \\
\hline Normal & 39 & 68,4 & 72 & 60 & 111 & 62,7 \\
\hline Sobrepeso & 13 & 22,8 & 37 & 30,9 & 50 & 28,3 \\
\hline Obesidad & 3 & 5,3 & 8 & 6,6 & 11 & 6,2 \\
\hline Activo & 29 & 50,9 & 23 & 19,2 & 52 & 29,4 \\
\hline Sedentario & 28 & 49,1 & 97 & 80,8 & 125 & 70,6 \\
\hline Antecedente familiar ECV & 29 & 50,9 & 45 & 37,5 & 74 & 41,8 \\
\hline Antecedente morbilidad ECV & 0 & 0 & 6 & 5,0 & 6 & 3,4 \\
\hline Tabaquismo & 34 & 50,6 & 46 & 38,3 & 80 & 45,1 \\
\hline $\mathrm{CC}$ & 22 & 38,6 & 72 & 60,0 & 94 & 53,1 \\
\hline Glicemia & 16 & 28,1 & 19 & 15,8 & 35 & 19,8 \\
\hline Col-total & 16 & 28,1 & 46 & 38,3 & 62 & 35,0 \\
\hline Triglicéridos & 6 & 10,5 & 3 & 2,5 & 9 & 5,1 \\
\hline $\mathrm{HDL}$ & 27 & 47,4 & 64 & 53,3 & 91 & 51,4 \\
\hline LDL & 23 & 40,4 & 55 & 45,8 & 78 & 44,1 \\
\hline Hipertensión Arterial & 4 & 7,0 & 4 & 3,3 & 8 & 4,5 \\
\hline Riesgo cardiovascular & 5 & 8,7 & 12 & 10,0 & 17 & 9,6 \\
\hline
\end{tabular}

TABLA 2

Factores de riesgo cardiovascular de los estudiantes según género.

\begin{tabular}{|c|c|c|c|}
\hline Factores de riesgo & Hombre & Mujer & $\mathrm{P}$ \\
\hline IMC (kg/mt²) & $24,06 \pm 3,23$ & $24,23 \pm 3,83$ & 0,868 \\
\hline Contorno de cintura (cm) & $87,37 \pm 12,13$ & $85,61 \pm 13,97$ & 0,202 \\
\hline HDL (mg/dl) & $40,80 \pm 6,79$ & $48,98 \pm 11,68$ & $0,000 *$ \\
\hline Col-total (mg/dl) & $186,98 \pm 31,92$ & $195,53 \pm 35,02$ & 0,133 \\
\hline Triglicéridos (mg/dl) & $95,13 \pm 50,09$ & $75,98 \pm 31,11$ & $0,035 *$ \\
\hline LDL (mg/dl) & $128,96 \pm 25,84$ & $131,19 \pm 28,29$ & 0,697 \\
\hline Glicemia (mg/dl) & $94,76 \pm 7,41$ & $92,17 \pm 9,62$ & $0,050 *$ \\
\hline Presión sistólica (mmHg) & $109,47 \pm 11,16$ & $104,20 \pm 13,82$ & $0,028 *$ \\
\hline Presión diastólica $(\mathrm{mmHg})$ & $67,63 \pm 7,26$ & $66,25 \pm 9,81$ & 0,392 \\
\hline
\end{tabular}


de estudiantes poseen riesgo cardiovascular (síndrome metabólico y/o resistencia a la insulina), prevalencia superior a la de estudiantes mexicanos que presentan 3,4\% (25). En Chile existe un aumento considerable de SM en menores de 25 años lo que es preocupante debido a que la Araucanía presenta la mayor prevalencia a nivel nacional con un 42\% (1). La presencia del SM no sólo aumenta el riesgo de desarrollar DM2 y ECV (26), sino también otras enfermedades crónicas (27), mientras que el manejo adecuado del SM modifica favorable y significativamente la evolución de cuadros patológicos crónicos asociados como diabetes y ateroesclerosis (28).

Al comparar variables antropométricas y metabólicas con presencia y no presencia de $S M$, se encontraron resultados similares en una población de adolescentes argentinos en IMC, contorno de cintura, HDL, glicemia y presión arterial (29), si bien poseen un rango etario menor, la identificación temprana es fundamental para reducir el riesgo de cardiopatía coronaria (30), además estos resultados muestran diferencias significativas al comparar entre hombres con mujeres en $\mathrm{HDL}$, TG y glicemia, asociaciones distintas a las descritas en estu- diantes brasileños donde únicamente existieron diferencias estadísticas en HDL (31).

Una investigación en universitarios españoles de primer año reportó diferencias significativas en la comparación del promedio de presión arterial sistólica entre hombres y mujeres (116,5 mm Hg y 124,5 mm Hg ) (32), concordantes a los presentes resultados, 109,47 y 104,20 mmHg en estudiantes varones y mujeres respectivamente, antecedentes a considerar, ya que la hipertensión arterial es uno de los principales factores de riesgo que contribuyen al aumento de la mortalidad (33).

El sobrepeso y la obesidad se asocian al desarrollo de insulino resistencia, diabetes tipo 2 y $\operatorname{ECV}(34,35)$. En este estudio 53,1\% de la población estudiada tenía obesidad abdominal, con una prevalencia de sobrepeso y obesidad de $28 \%$ y $6 \%$ respectivamente, similares a los hallazgos descritos en una población universitaria (36) y con un promedio del IMC levemente mayor en mujeres, resultados concordantes con estudiantes universitarios americanos (37). El promedio de contorno de cintura tuvo un valor mayor tanto en hombres como en mujeres a los descritos en otra población de educa-

\section{TABLA 3}

Factores de riesgo en los estudiantes según presencia o ausencia de síndrome metabólico.

\begin{tabular}{|c|c|c|c|}
\hline Factores de riesgo & Con SM & $\operatorname{Sin} S M$ & $\mathrm{P}$ \\
\hline IMC (kg/mt²) & $27,91 \pm 4,34$ & $23,80 \pm 3,43$ & $0,000 *$ \\
\hline Contorno de cintura $(\mathrm{cm})$ & $93,23 \pm 7,46$ & $84,11 \pm 9,54$ & $0,002 *$ \\
\hline $\mathrm{HDL}(\mathrm{mg} / \mathrm{dl})$ & $40,06 \pm 7,69$ & $46,86 \pm 11,11$ & $0,028 *$ \\
\hline Col-total (mg/dl) & $194,74 \pm 37,99$ & $192,60 \pm 33,99$ & 0,835 \\
\hline Triglicéridos (mg/dl) & $67,71 \pm 20,56$ & $74,99 \pm 26,08$ & 0,536 \\
\hline $\mathrm{LDL}(\mathrm{mg} / \mathrm{dl})$ & $129,22 \pm 28,53$ & $130,57 \pm 27,47$ & 0,865 \\
\hline Glicemia (mg/dl) & $98,17 \pm 7,48$ & $92,64 \pm 7,85$ & $0,020 *$ \\
\hline Presión sistólica (mmHg) & $119,23 \pm 16,43$ & $104,84 \pm 12,43$ & $0,002 *$ \\
\hline Presión diastólica (mmHg) & $73,46 \pm 9,43$ & $66,14 \pm 8,88$ & 0,006 * \\
\hline
\end{tabular}

\section{TABLA 4}

Factores de riesgo cardiovascular en los estudiantes, según actividad física.

\begin{tabular}{|c|c|c|c|}
\hline Factores de riesgo & Activo & Sedentario & $\mathrm{P}$ \\
\hline $\mathrm{IMC}\left(\mathrm{kg} / \mathrm{mt}^{2}\right)$ & $23,94 \pm 3,15$ & $24,28 \pm 3,84$ & 0,658 \\
\hline Contorno de cintura $(\mathrm{cm})$ & $84,8 \pm 9,33$ & $86,75 \pm 14,77$ & 0.919 \\
\hline $\mathrm{HDL}(\mathrm{mg} / \mathrm{dl})$ & $43,28 \pm 10,49$ & $47,54 \pm 11,04$ & $0,016 *$ \\
\hline Col-total (mg/dl) & $183,45 \pm 29,45$ & $196,44 \pm 35,33$ & $0,027 *$ \\
\hline Triglicéridos (mg/dl) & $82,9 \pm 37,02$ & $81,89 \pm 40,15$ & 0,929 \\
\hline LDL (mg/dl) & $125,68 \pm 22,3$ & $132,36 \pm 29,12$ & 0,206 \\
\hline Glicemia (mg/dl) & $93,88 \pm 7,01$ & $92,66 \pm 9,71$ & 0,388 \\
\hline Presión sistólica (mmHg) & $106,5412,027$ & $105,64 \pm 13,737$ & 0,895 \\
\hline Presión diastólica(mmHg) & $66,547,576$ & $66,76 \pm 9,657$ & 0,925 \\
\hline
\end{tabular}


ción superior (38).

La hipercolesterolemia se presentó en 35\% de los estudiantes. A pesar de tratarse de una población joven. Otros parámetros lipídicos también se observaron aumentados: así $51,4 \%$ presentó valores disminuidos de HDL y $44,1 \%$ niveles de LDL sobre lo normal, resultados superiores a los reportados en una investigación con una muestra de estudiantes, donde el Col-total y LDL fue $9,7 \%$ y 5,9\% respectivamente, en tanto, el HDL se mostró disminuido en 12\% (39).

El $45,1 \%$ de los estudiantes fumaba, misma tendencia $(40,6 \%)$ se observó en la población general adulta chilena (1), pero valores superiores a lo reportado en otro grupo de estudiantes universitarios chilenos (32,8\%) (40). El consumo de tabaco es un factor de riesgo potente para el desarrollo de enfermedades, incluyendo el cáncer oral, enfermedades cardiovasculares y pulmonares y con un marcado efecto perjudicial en la salud de la población (41).

Un 3,4\% de los estudiantes presentó antecedentes de morbilidad asociados a ECV, todos correspondían a mujeres con diagnóstico de resistencia a la insulina. Se estima que en aquellas personas con antecedentes familiares de ECV, el riesgo de padecer un evento coronario es aproximadamente el doble con respecto a quienes no presentan estos antecedentes (42).

La investigación demostró la presencia de FR de ECV en una población joven, donde destaca la prevalencia de malnutrición por exceso, obesidad abdominal, sedentarismo, tabaquismo, hipercolesterolemia y, en menor medida, presencia de SM. A pesar de la asociación entre la práctica de AF y la reducción de ECV (43), los niveles de AF permanecen bajos en la población (44), y a través de esto podemos estimar que a mediano plazo estaremos ante un aumento importante de personas jóvenes con riesgo cardiovascular en la Región de la Araucanía.

\section{RESUMEN}

Introducción: Las enfermedades crónicas no transmisibles (ECNT) incrementan la presencia de factores de riesgo (FR) de enfermedad cardiovascular (ECV) en jóvenes. Objetivo: Analizar la relación entre estado nutricional (EN), actividad física (AF) y prevalencia de FR cardiovascular en estudiantes de la Universidad Santo Tomás sede Temuco, ingreso 2010. Sujetos y métodos: Se realizó un estudio descriptivo de corte trasversal; de un total de 348 estudiantes, se obtuvo una muestra de 177 sujetos, de los cuales 32,2\% correspondió

Tabla 5

Factores de riesgo cardiovascular en los estudiantes según circunferencia de cintura.

\begin{tabular}{|c|c|c|c|}
\hline Factores de riesgo & Normal & Alto & $\mathrm{P}$ \\
\hline $\mathrm{IMC}\left(\mathrm{kg} / \mathrm{mt}^{2}\right)$ & $22,1 \pm 2,52$ & $26,0 \pm 3,5$ & $0,000 *$ \\
\hline $\mathrm{HDL}(\mathrm{mg} / \mathrm{dl})$ & $46,7 \pm 11,5$ & $46,0 \pm 10,7$ & 0,739 \\
\hline Col- total $(\mathrm{mg} / \mathrm{dl})$ & $186,1 \pm 33,9$ & $198,7 \pm 33,5$ & $0,012 *$ \\
\hline Triglicéridos (mg/dl) & $71,8 \pm 24,0$ & $77,4 \pm 27,4$ & 0,178 \\
\hline $\mathrm{LDL}(\mathrm{mg} / \mathrm{dl})$ & $125,6 \pm 25,7$ & $134,7 \pm 28,4$ & $0,032 *$ \\
\hline Glicemia (mg/dl) & $94,6 \pm 8,4$ & $91,7 \pm 7,4$ & $0,022 *$ \\
\hline Presión sistólica (mm/Hg) & $103,98 \pm 14,873$ & $107,61 \pm 11,400$ & 0,066 \\
\hline Presión diastólica $(\mathrm{mm} / \mathrm{Hg})$ & $65,42 \pm 9,279$ & $67,82 \pm 8,785$ & 0,030 * \\
\hline
\end{tabular}

\section{TABLA 6}

Factores de riesgo en los escolares según estado nutricional.

\begin{tabular}{|c|c|c|c|c|c|}
\hline Factores de riesgo & Enflaquecido & Normal & Sobrepeso & Obesidad & $\mathrm{P}$ \\
\hline Contorno de cintura (cm) & $74,6 \pm 10,74$ & $81,19 \pm 7,24$ & $94,22 \pm 15,09$ & $105,38 \pm 18,23$ & $0,000 *$ \\
\hline HDL (mg/dl) & $51,76 \pm 16,5$ & $47,75 \pm 11,45$ & $42,88 \pm 9,27$ & $43,9 \pm 7,69$ & 0,056 \\
\hline Col-total (mg/dl) & $182,02 \pm 35,91$ & $189,69 \pm 33,67$ & $195,51 \pm 33,1$ & $215,11 \pm 36,87$ & 0,079 \\
\hline Triglicéridos (mg/dl) & $62,6 \pm 10,92$ & $78 \pm 31,64$ & $86,52 \pm 39,68$ & $112,33 \pm 78,44$ & 0,713 \\
\hline LDL(mg/dl) & $117,74 \pm 30,54$ & $127,08 \pm 24,56$ & $135,33 \pm 31,44$ & $148,75 \pm 28,68$ & $0,028 *$ \\
\hline Glicemia (mg/dl) & $89,8 \pm 7,16$ & $92,49 \pm 8,99$ & $93,7 \pm 10,05$ & $96,5 \pm 4,68$ & 0,361 \\
\hline Presión sistólica (mmHg) & $105,0 \pm 8,66$ & $103,97 \pm 13,62$ & $108,23 \pm 11,55$ & $115,00 \pm 13,31$ & $0,013 *$ \\
\hline Presión diastólica $(\mathrm{mmHg})$ & $64,00 \pm 8,94$ & $65,36 \pm 9,36$ & $68,96 \pm 8,37$ & $71,25 \pm 6,07$ & $0,016 *$ \\
\hline
\end{tabular}

Datos presentados como media \pm Desviación estándar. HDL: colesterol HDL. Col-total: Colesterol total. LDL: Colesterol LDL.

Para la comparación de las variables paramétricas entre más de dos grupos se utilizó ANOVA y en el caso de variables no paramétricas, se utilizó la prueba de la H de Kruskal- Wallis. * Existe significancia estadística $p<0,05$. 
a hombres y $68,8 \%$ a mujeres, la edad fluctuó entre 18 y 25 años. Se realizaron mediciones antropométricas, de presión arterial, muestras sanguíneas, encuestas de AF y antecedentes de morbilidad. Los resultados permitieron estimar el EN, la práctica de AF y la prevalencia de los distintos FR. Resultados: El estudio reveló que $34,5 \%$ de los estudiantes presentaban tiene malnutrición por exceso, 70,6\% eran sedentarios y $9,6 \%$ tenían riesgo de enfermedad cardiovascular por presencia de Síndrome Metabólico (SM); mientras, el riesgo por obesidad abdominal fue de 53,1\%. Los parámetros que evidenciaron diferencias significativas fueron el colesterol HDL que se mostró disminuido en los estudiantes activos $(p=0,016)$ y el colesterol total, que se observó levemente aumentado en los sedentarios $(p=0,027)$. Al analizar la AF con las variables IMC, triglicéridos, colesterol LDL, glicemia y presión arterial, no se observaron diferencias significativas. Conclusiones: Se observó alta prevalencia de malnutrición por exceso, sedentarismo y factor de riesgo de ECV con predominio en las mujeres.

Palabras clave: Factores de riesgo; enfermedades cardiovasculares; obesidad; estudiantes.

\section{BIBLIOGRAFÍA}

1. MINSAL. Encuesta Nacional de Salud 2009-2010. Ministerio de Salud, 2010.

2. Zímmermann M, González M, Labaca I. Perfiles de exposición de riesgo cardiovascular según la ocupación laboral en la Comunidad de Madrid. Rev Esp Salud Pública. 2010; 84:293-308.

3. Pencina MJ, D'Agostino RB, Larson MG, Massaro JM, Vasan RS. Predicting the 30-Year Risk of Cardiovascular Disease. Circulation 2009; 119 (24): 3078-84

4. OMS. Informe sobre la salud en el mundo. 2013 [up-dated 2014; cited 2014 Noviembre ]; Disponible en: http:// www.who.int/publications/en/.

5. WHO. World Health Organization. The World Health Report 2002. Reducing Risks, Promoting Healthy Life. WHO 2002. Disponible en: http://www.who.int/whr/2002/en/ whrO2_en.pdf

6. Bustos P, Amigo H, Arteaga A, Agosta AM, Roña RJ. Factores de riesgo de enfermedad cardiovascular en adultos jóvenes. Rev Méd Chil. 2003; 131: 973-80.

7. Villalón G, Vera S. Evolución de la mortalidad en Chile según causas de muerte y edad: 1990-2007. Publicación Especial. Chile: Instituto Nacional de Estadística; 2010.

8. World Health Organization. Preventing chronic diseases: WHO global report. Genova, Suiza: WHO, 2005.

9. Instituto Nacional de Estadísticas, Proyecciones y Estimaciones de Población, Total País 2004. Disponible en: http://deis.minsal.cl/deis/ev/esperanza_de_vida/esperanza_de_vida_por_quinquenios_sexo.htm

10. Palomo I, Torres G, Alarcón M, Maragaño P, Leiva E, Mujica $V$. Alta prevalencia de factores de riesgo cardiovascular clásicos en una población de estudiantes universitarios de la región centro-sur de Chile. Rev Esp Cardiol. 2006; 59: 1099-10.

11. Cecchini M, Sassi F, Lauer J, Lee Y, Guajardo-Barrón C. Tackling of unhealthy diets, physical inactivity, and obesity: health effects and cost- effectiveness. Lancet. 2010; 376:1775- 84

12. Bustos $P$, Amigo H, Vásquez A, Vargas C. Evolución del síndrome metabólico y de sus componentes en un seguimiento de 10 años en adultos de la Región de Valparaíso. Rev Med Chil. 2014; 142( 5 ): 579-86.

13. Morales $G$, del Valle $C$, Soto $A$, Ivanovic D. Factores de riesgo cardiovascular en estudiantes universitarios. Rev Chil Nutr. 2013; 40( 4 ): 391-6.

14. Ibrahim N, Mahnashi M, Al-Dhaheri A, Al-Zahrani B, AlWadie E, Aljabri M, Al-Shanketi R, Al-Shehri R, Al-Sayes F, Bashawri J. Risk factors of coronary heart disease among medical students in King Abdulaziz University, Jeddah, Saudi Arabia. BMC Public Health. 2014;14:411.

15. Fernandes J, Arts J, Dimond E, Hirshberg S, Lofgren IE. Dietary factors are associated with coronary heart disease risk factors in college students. Nutr Res. 2013; (8):647-52.

16. Kutlu R., Erdem R. Evaluation of cardiovascular risk factors among university students in Turkey: a cross-sectional survey. Russian Open Med J. 2013; 2: 37.

17. MINSAL, Ministerio de Salud, Gobierno de Chile. Departamento de Epidemiología. Departamento de Promoción de Salud. II Encuesta Nacional de Calidad de Vida y Salud. Chile 2006. Cuestionario. Disponible en: http://epi.minsal. cl/epi/html/sdesalud/calidaddevida2006/cuestionario.pdf

18. MINSAL, Ministerio de Salud. Guía clínica hipertensión arterial primaria o esencial en personas de 15 años y más. Santiago: 2010. Disponible en: http://web.minsal.cl/portal/url/item/73b3fce9826410bae04001011f017f7b.pdf

19. Martínez M, Leiva A., Sotomayor C, Victoriano T, Von Chrismar A, Pineda S. Factores de riesgo cardiovascular en estudiantes de la Universidad Austral de Chile. Rev Med Chil. 2012; 140(4): 426-35.

20. Macías M, Pérez V, Rivera A, Martínez M, Ramírez J, Kornhauser C. Physical activity and cardiovascular risk factors in university students in the city of Leon, México. Health. 2013 (5) 1861-5.

21. Bowden, R. G., Lanning, B. A., Doyle, E. I., Johnston, H. M., Slonaker, G. S., \& Scanes, G. Lipid levels in a cohort of sedentary university students. Internet J Cardiovasc Res. 2005;2 (2).

22. Warburton DE, Nicol CW, Bredin SS. Health benefits of physical activity: the evidence. Can Med Assoc J. 2006; 174: 801-9.

23. Proper KI, Singh AS, van-Mechelen W, Chinapaw MJM. Sedentary Behaviors and Health Outcomes Among Adults. A Systematic Review of Prospective Studies. Am J Prev Med. 2011; 40: 174-82.

24. Gibala M, Little J, MacDonald M, Hawley J. Physiolo gical adaptations to low-volume high intensity interval training in health and disease. J Physiol. 2012; 590 : 1077-84.

25. González E, Palmeros C, Villanueva J, Torres B, Bastida $S$, Vaquero M, Sánchez F. Metabolic syndrome prevalence and its association with the body mass index in university students Med Clin (Barc). 2007;129(20):766-9.

26. Grundy SM, Cleeman JI, Daniels SR, Donato KA, Eckel RH, Franklin BA. Diagnosis and Management of the Metabolic Syndrome: An American Heart Association/National Heart Lung and Blood Institute Scientific Statement. Circulation. 2005; 112: 285-90.

27. von-Bernhardi $R$, Zanlungo S, Arrese M, Arteaga A, Rigotti $A$. El síndrome metabólico: De factor agravante a principal factor de riesgo patogénico en diversas enfermedades crónicas. Rev Med Chil. 2010; 138: 1012-9.

28. Early J. Comprehensive management of cardiometabolic risk factors. Clin Cornerstone 2007; 8: 69-80.

29. Graffigna M.N, Honfi M, Soutelo J, Migliano M, Ledesma $L$, Proietti A, Aranguren M, Pazos M, Musso C, Berg G. Metabolic syndrome and cardiovascular risk factors in adolescent students from Buenos Aires Rev Argent Endocrinol Metab. 2010; 47: 14-20. 
30. Fernandes J, Lofgren IE. Prevalence of metabolic syndrome and individual criteria in college students. J Am Coll Health. 2011;59(4):313-21.

31. Costa Silva ZJ, Barreto CL, De Castro CL, Duarte PG, Toyomi $H A$ and Sachs A. Lipid profile and cardiovascular risk factors among first-year Brazilian university students in São Paulo. Nutr Hosp. 2011; 26: 553-9.

32. Irazusta $A$, Itziar $H$, Irazusta J, Ruiz F, Díaz E, Gil J. Increased cardiovascular risk associated with poor nutritional habits in first-year university students. J Nutr Res. 2007; (27) 387-94.

33. Pyle MA, Lalumandier JA, Sawyer DR. Prevalence of elevated blood pressure in students attending a college oral health program. Spec Care Dentist. 2000;20(6):234-9.

34. Celis $C$, Pérez F, Ibanes $L$, Sanzana R, Hormazabal E, Ulloa $N$, et al. Insulin resistance in Chileans of European and indigenous descent: evidence for an ethnicity $x$ environment interaction. PloS One. 2011; 6 (9): e24690.

35. McLaughlin T, Allison G, Abbasi F, Lamendola C, Reaven $G$. Prevalence of insulin resistance and associated cardiovascular disease risk factors among normal weight, overweight, and obese individuals. Metabolism. 2004; 53 (4): 495-9.

36. Abdel-Megeid FY, Abdelkarem HM, El-Fetouh AM. Unhealthy nutritional habits in university students are a risk factor for cardiovascular diseases. Saudi Med J. 2011;32(6):621-7.

37. Topè AM, Rogers PF. Metabolic syndrome among students attending a historically black college: prevalence and gender differences. Diabetol Metab Syndr. 2013; 12;5(1):2.

38. Yeasmin R, Nahar N.,Begum K, Parveen S, Akhter F, Aziz M, Islam F, Eyaub T. Lipid Profile, Glycaemic and Anthropometric Status of Students of a Private Medical College in Dhaka City. AKMMC J 2014; 5(1): 23-8.

39. Freitas $R$, Araújo M, Lima A, Pereira D, Alencar A, Damasceno M.. Análisis del perfil lipídico en una población de estudiantes universitarios. Rev Latino-Am. Enfermagem. 2013;21(5).

40. Morales G, Del Valle C, Belmar C, Orellana Y, Soto A, Ivanovic $D$. Prevalencia de consumo de drogas en estudiantes universitarios que cursan primer y cuarto año. Rev Med Chil. 2011; 139: 1573-80.

41. Paul $Y$, Soni $N$, Vaid $R$, Basavaraj $P$, Khuller $N$. IIl effects of smoking on general and oral health: Awareness among college going students. J Indian Assoc Public Health Dent. $2014 ; 12: 47-53$.

42. Sesso, H, Lee I, Gaziano J, Rexrode K., Glynn R., Buring J. Maternal and paternalhistory of myocardial infarction and risk of cardiovascular disease in men and women. Circulation. 2001; 24;104 (4):393-8.

43. Ahmed HM, Blaha MJ, Nasir K, Rivera JJ, Blumenthal RS. Effects of physical activity on cardiovascular disease. Am J Cardiol. 2012; 109 (2): 288-95.

44. Dumith SC, Hallal PC, Reis RS, Kohl HW, 3rd. Worldwide prevalence of physical inactivity and its association with human development index in 76 countries. Prev Med. 2011; 53 (1-2): 24-8. 